\title{
Concomitant Medication Adverse Event Number
}

National Cancer Institute

\section{Source}

National Cancer Institute. Concomitant Medication Adverse Event Number. NCI

Thesaurus. Code C83097.

The numerical identifier of the adverse event associated with the concomitant

medication. 